\title{
Clique Matrix of a Graph in Traffic Control Problems
}

\author{
Arun Kumar Baruah \\ Department of Mathematics \\ Dibrugarh University \\ Dibrugarh - 786004, Assam, India
}

\author{
Niky Baruah \\ Department of Mathematics \\ Dibrugarh University \\ Dibrugarh - 786004, Assam, India
}

\begin{abstract}
Clique matrix can be used as a graph theoretic tool to study traffic control problem at an intersection. A traffic control problem can be efficiently modelled as a graph where the nodes represent the traffic streams and the edges represent the relationship among the streams. The matrix representation of the control problem is used for phasing of signal groups and thus providing a solution to the control problem. Clique of a graph is defined as maximal complete subgraph and clique matrix of a graph is defined as a generalization of the incidence matrix where the columns correspond to the number of cliques and the rows correspond to the number of vertices of the graph.
\end{abstract}

\section{Keywords}

Clique Matrix, Cycle Time, Signal Group, Traffic Control, Traffic Streams.

\section{INTRODUCTION}

Transportation has always been a crucial aspect of human civilization, but as vehicular traffic began to increase, the congestion on the streets began to hamper the safe and efficient movement of traffic [1], [2]. More and more accidents were caused and serious problems of parking and environmental pollution began to increase and therefore it is necessary to give increasing attention to the operational characteristic of transportation and study the need for better geometric design, capacity, intersection, traffic regulation, signals etc. Due to the increase in general traffic volumes, the road network is subjected to ever increasing demands. Since traffic signals are used to control large number of junctions, the achievement of good control performance at signal controlled road junctions is now of great importance. The problem of designing a control scheme for a junction or intersection can be divided into two parts. The streams of traffic and pedestrian movements are first identified and divided into mutually compatible sets which are to receive right- of-way together and an order is to be determined in which right-of-way is to be granted to these sets [3]. Once this has been done, suitable durations are chosen for the periods during which each of the sets receives right-of-way.

Although complete subgraph have been studied for longer in Mathematics, the term clique came from Social Sciences where complete subgraphs are used to study social cliques, i.e. groups of people who all know each other. The clique terminology comes from Luce and Perry in 1949 who were motivated by sociological applications.

Various algorithms for finding cliques of graphs were developed by Bierstone and Mulligan and presented in the analysis of clustering techniques by Auguston and Minker [8]. Coen Bron and Joep Kerboscht presented two versions of backtracking algorithms, using branch and bound techniques to find the number of cliques in an undirected graph [9], [10].
The vehicular arrivals at a junction on a single approach may be considered to form one or more streams. Vehicles forms a particular stream is assumed to queue together and to receive identical signal indication. Signal control at a junction is granting period of right-of- way to streams of traffic or pedestrians wishing to use the intersection. Traffic congestion leads to queuing phenomenon and corresponding delays while the infrastructure capacity is fully utilized. In the worst case, it may lead to a degraded use of the available infrastructure thus contributing to an accelerated congestion increase. Thus traffic congestion results in excess delay, reduced safety and increase environmental pollution.

The efficient, safe and less pollution transportation of persons and goods calls for an optimal utilization of the available infrastructure via suitable application of a variety of traffic control measures. This trend is enabling by the rapid developments in the areas of communications and technologies, but the efficiency of traffic control directly depends on the efficiency and relevance of employed control methodologies [4].

In this paper, we have used clique matrix of an undirected graph [5], as a graph theoretic tool to study traffic control problem at a signalized intersection and shown that the Clique matrix can be used to give an optimal solution of a control problem at a larger intersection, where the number of cliques formed are more.

\section{CLIQUE OF A GRAPH}

By a graph $\boldsymbol{G}=(\boldsymbol{V}, \boldsymbol{E})$ we mean a finite undirected graph without loops or multiple edges, where $\boldsymbol{V}$ is the set of vertices and $\boldsymbol{E}$ is the set of edges. A clique of a graph $\boldsymbol{G}$ is a maximal complete subgraph of $\boldsymbol{G}$ [6], [7].

Alternatively, a clique can also be defined as follows:

A subset $S$ of vertices satisfying the following properties:

- $\quad$ S contains three or more vertices.

- Each pair of vertices in S has an edge connecting them.

- $\quad \mathrm{S}$ is maximal (there is no larger set of vertices that satisfies the second property and contains $\mathrm{S}$ )

\section{CLIQUE MATRIX OF A GRAPH}

We define a clique matrix $\boldsymbol{C}=\left[\boldsymbol{c}_{\boldsymbol{i}}\right]$ of a graph in which the rows correspond to the vertices of the graph and the column to the number of cliques formed :

$$
\begin{aligned}
\boldsymbol{c}_{\boldsymbol{i} \boldsymbol{j}} & =1 ; \text { if } \boldsymbol{i}^{\boldsymbol{t h}} \text { clique contains } \boldsymbol{j}^{\boldsymbol{t h}} \text { vertex } \\
& =0 ; \text { otherwise. }
\end{aligned}
$$

For example, let us consider a graph with five vertices and eight edges as shown in the Fig. 1 below:

The cliques formed are : 


$$
\begin{aligned}
& C_{1}=\left\{v_{1}, v_{2}, v_{5}\right\} \\
& C_{2}=\left\{v_{1}, v_{4}, v_{5}\right\} \\
& C_{3}=\left\{v_{1}, v_{2}, v_{4}\right\} \\
& C_{4}=\left\{v_{2}, v_{3}, v_{4}\right\} \\
& C_{5}=\left\{v_{2}, v_{4}, v_{5}\right\} \\
& C_{6}=\left\{v_{1}, v_{2}, v_{4}, v_{5}\right\}
\end{aligned}
$$

The clique matrix formed by the definition is :

$$
\boldsymbol{C}=\left[\begin{array}{llllll}
1 & 1 & 1 & 0 & 0 & 1 \\
1 & 0 & 1 & 1 & 1 & 1 \\
0 & 0 & 0 & 1 & 0 & 0 \\
0 & 1 & 1 & 1 & 1 & 1 \\
1 & 1 & 0 & 0 & 1 & 1
\end{array}\right]
$$

\section{Observations:}

From the clique matrix the following observations can be made :

1. The number of ones in each row represents the number of cliques formed by the vertex.

2. The number of ones in each column represents the size of the clique i.e. whether it is 2-vertex clique, 3 -vertex clique etc.
3. A row with all the entries zero corresponds to a non clique vertex i.e. a weakly connected vertex and does not form clique with any other vertex.

Although a pictorial representation of a graph is very convenient, matrix representation is also a better and convenient way of representing a graph useful for computing process. Matrices are easy for manipulation and can be readily applied to study the structural properties of graph from an algebraic point of view. In many applications of graph theory, such as electrical network, control problems and operational research, matrix also turn out to be the natural way of expressing the problem.

The clique matrix is the generalization of the incidence matrix as the incidence matrix is also a clique matrix where the cliques formed is the number of edges corresponding to the number of vertices. We introduce the terminology clique matrix for the incidence matrix since each column express which vertex form a clique. The incidence matrix is a special case, namely a 2 -vertex clique matrix. The number of columns in the clique matrix is the number of cliques formed and each column describes the clique either it is 2- vertex clique, 3vertex clique or more.

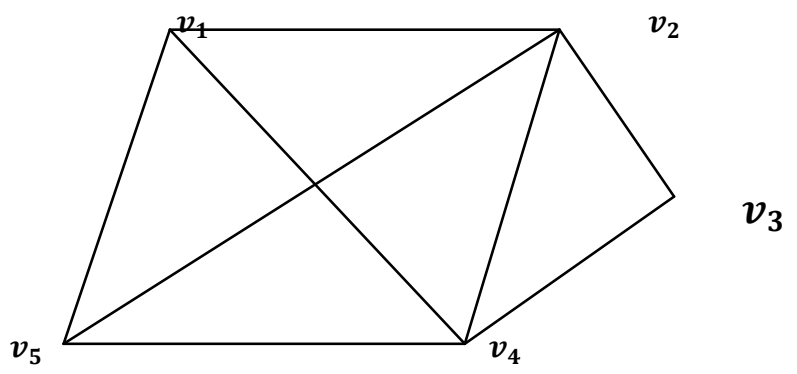

Fig. 1 : Graph with five vertices

\section{FORMULATION OF THE PROBLEM}

A traffic control problem at an intersection can be modelled by a graph where the nodes represent the traffic streams and the edges represent the relationship among the streams. The traffic streams which can simultaneously move together will be joined by an edge and the streams which are in a conflict there will be no edge connecting them.
Let us consider a traffic control problem at an intersection with five streams $\sigma_{1}, \sigma_{2}, \sigma_{3}, \sigma_{4}$ and $\sigma_{5}$ together with the direction as shown in the Fig. 2 below. The streams which can simultaneously move together will be joined by an edge and the set of streams which can move through the intersection without any conflict i.e. the cliques formed of the corresponding graph of the traffic control problem. In this paper we shall use clique matrix as a graph theoretic tool for controlling the flow of traffic at an intersection. The graph representing the said intersection is as shown in the figure Fig. 3 : 

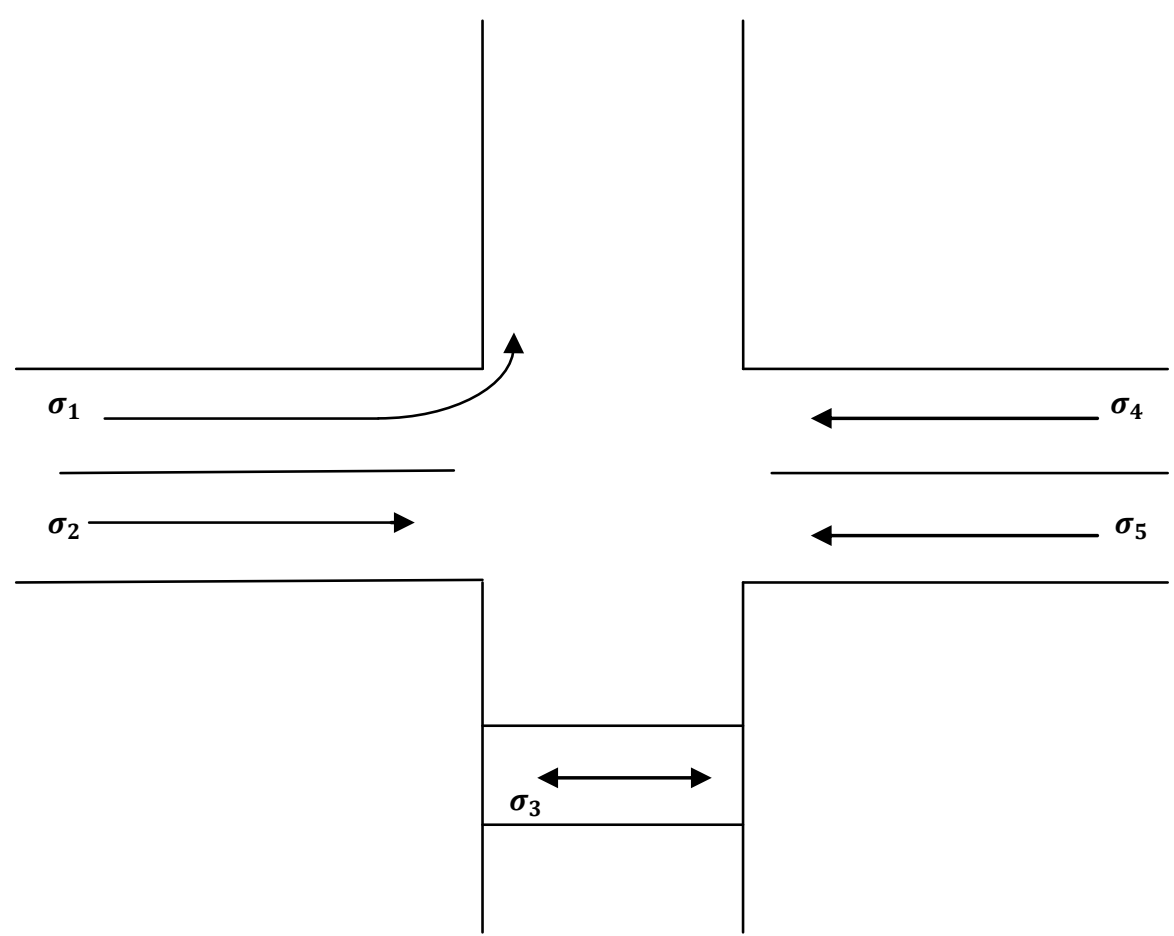

Fig. 2 : An Intersection with five streams

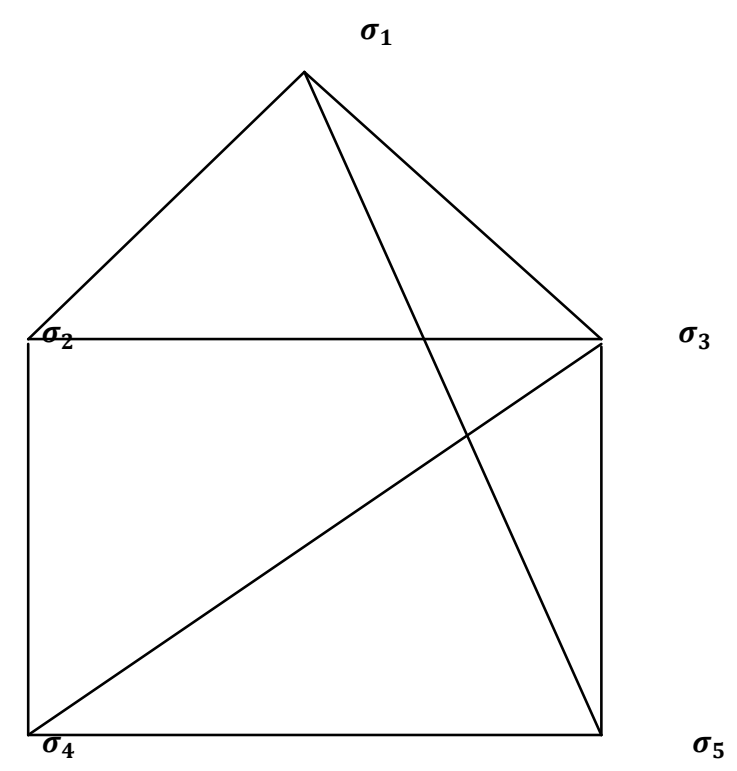

Fig. 3 : A graph representing the above intersection (Fig. 2)

According to the definition of clique matrix the numbers of cliques formed are:

$$
\begin{aligned}
& C_{1}=\left\{\sigma_{1}, \sigma_{2}, \sigma_{3}\right\} \\
& C_{2}=\left\{\sigma_{2}, \sigma_{3}, \sigma_{4}\right\} \\
& C_{3}=\left\{\sigma_{3}, \sigma_{4}, \sigma_{5}\right\} \\
& C_{4}=\left\{\sigma_{1}, \sigma_{3}, \sigma_{5}\right\}
\end{aligned}
$$

The matrix representation of the graph

$$
\mathbf{C}=\left[\begin{array}{llll}
1 & 0 & 0 & 1 \\
1 & 1 & 0 & 0 \\
1 & 1 & 1 & 1 \\
0 & 1 & 1 & 0 \\
0 & 0 & 1 & 1
\end{array}\right]
$$

As the number of columns represents the cliques formed by the graph, which also depicts the number of 
solutions that can be obtained from the graph. Here the numbers of cliques formed are four and hence phasing can be done accordingly and cycle time can be divided depending on the number of solutions. As clique of a graph represents the maximum connected subgraph, it is therefore necessary to find the maximum number of traffic streams that can simultaneously move together through the intersection that can be found from the clique matrix, thus forming a solution to the control problem.

In the clique matrix a row with all the entries one represents a strongly connected node and it form cliques with all other vertices of the graph. Here in this example the vertex $\sigma_{3}$ is compatible with all other streams and hence can be allowed to move simultaneously through the intersection with the other streams at the same time. On the other hand, a row with all the entries zero represents a node that does not form clique i.e. is not compatible with other streams of the intersection and have to be controlled independently by allotting different cycle time.

For a larger intersection where the numbers of cliques formed are more and consequently the number of cliques formed is more, the clique matrix can be used for grouping or phasing of signal groups. As clique of a compatible graph represents a solution to the problem, the cliques can be divided into set of signal groups which can simultaneously move together through the intersection. From the clique matrix it can be easily found that the streams which are common to most of the cliques can be put in one signal group and phasing can be done. In some cases it may be necessary to control traffic in such a way that certain streams can pass through an intersection without any conflict i.e. it is necessary to find the set of conflicting traffic streams that can simultaneously get the right-of-way and can be put in one signal group. For a larger intersection, the numbers of streams are more resulting in the formation of large number of cliques. Here it is not possible to allow each of them to move once in the cycle which is also the trivial solution. Therefore some amount of conflict can be permitted to obtain a better solution which can be done from the clique matrix without going back to the intersection. Thus for a traffic control problem at an intersection, the clique matrix depicts the solution or the control of traffic at the particular intersection as the number of cliques formed represents a solution to the control problem.

\section{PHASING OF SIGNAL GROUP}

Signal Group is defined as the set of traffic streams which get identical signal indication at an intersection [11]. For the traffic control problem of the previous section the cliques formed $\boldsymbol{C}_{1}, \boldsymbol{C}_{2}, \boldsymbol{C}_{3}$ and $\boldsymbol{C}_{\mathbf{4}}$ are nothing but the signal groups which can simultaneously move through the intersection with any conflict. If we consider the cycle time to be $\mathbf{6 0}$ seconds, each can be allowed to move through the intersection once i.e. for $\mathbf{1 5}$ seconds which is also the trivial solution.

But a better solution can be obtained from the clique matrix. It is observed from the clique matrix that the cliques in which most of the streams are common can be put in one signal group. In the above intersection, the cliques $\boldsymbol{C}_{\mathbf{1}}$ and $\boldsymbol{C}_{\mathbf{2}}$ can be put in one signal group as the streams $\boldsymbol{\sigma}_{2}$ and $\boldsymbol{\sigma}_{3}$ are common and hence can be allowed to move simultaneously, although the streams $\sigma_{1}$ and $\sigma_{4}$ conflicts it can be allowed to move simultaneously through the intersection as the left turn of the traffic stream $\sigma_{1}$ can be allowed with $\sigma_{4}$. Also $\boldsymbol{C}_{3}$ and $\boldsymbol{C}_{4}$ can be allowed to move together in the same interval as $\boldsymbol{\sigma}_{3}$ and $\boldsymbol{\sigma}_{\mathbf{5}}$ are common to both the cliques. Let us denote the set of signal groups as $\boldsymbol{S}_{\mathbf{1}}$ and $\boldsymbol{S}_{\mathbf{2}}$ :

$$
\begin{aligned}
S_{1} & =\left\{\left\{\sigma_{1}, \sigma_{2}, \sigma_{3}\right\},\left\{\sigma_{2}, \sigma_{3}, \sigma_{4}\right\}\right\} \\
& =\left\{C_{1}, C_{2}\right\} \\
S_{2} & =\left\{\left\{\sigma_{3}, \sigma_{4}, \sigma_{5}\right\},\left\{\sigma_{1}, \sigma_{3}, \sigma_{5}\right\}\right\} \\
& =\left\{C_{3}, C_{4}\right\}
\end{aligned}
$$

This solution is better as compared to the earlier one as most of the streams are moving except $\boldsymbol{\sigma}_{\mathbf{5}}$ in $\boldsymbol{S}_{\mathbf{1}}$ and $\boldsymbol{\sigma}_{\mathbf{2}}$ in $\boldsymbol{S}_{\mathbf{2}}$. Considering the cycle time to be $\mathbf{6 0}$ seconds, $\boldsymbol{S}_{\mathbf{1}}$ and $\boldsymbol{S}_{\mathbf{2}}$ can be allowed to move for $\mathbf{3 0}$ seconds each. The diagrammatic representations of the two cases are shown in the Fig. 4 and in the Fig. 5 respectively.

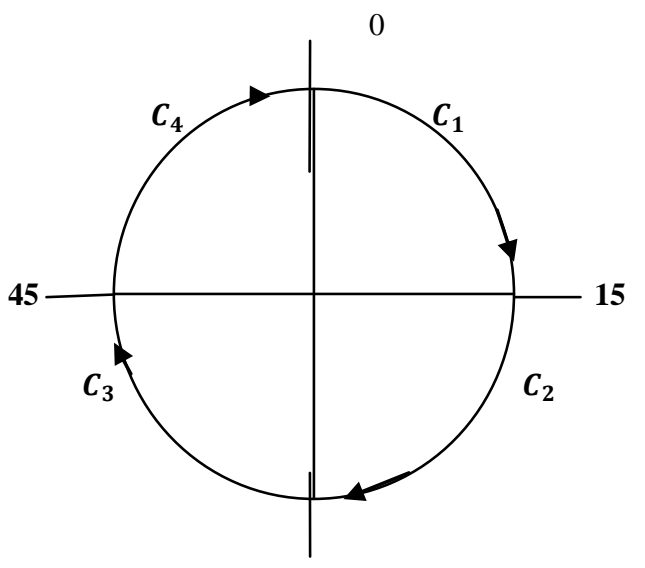

30

\section{0 - 15 seconds $C_{1}$ moves}

15 - 30 seconds $C_{2}$ moves

\section{$30-45$ seconds $C_{3}$ moves}

45 - 60 seconds $C_{4}$ moves

Fig. 4 : Cycle Time : 15 Seconds 


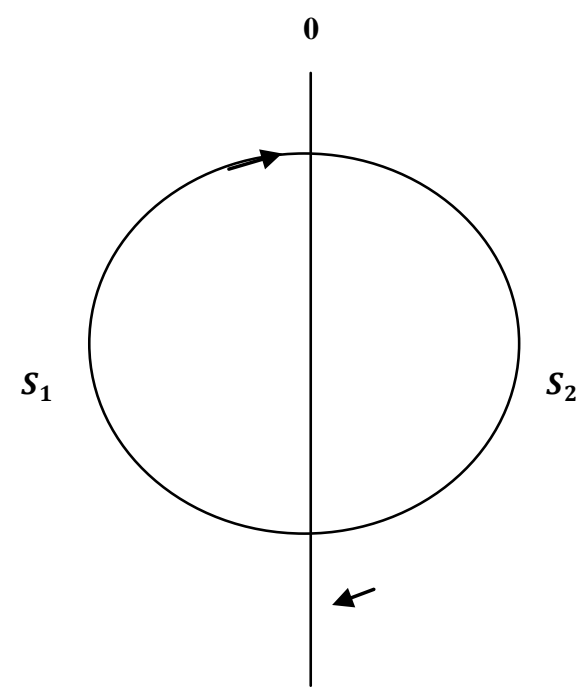

\section{APPLICATION OF CLIQUE MATRIX}

Clique Matrix has many other applications in Science and particular in Social Sciences to study the interpersonal relationship among a group of people [12]. The problems of Social Sciences are : we consider any binary interpersonal relationship among members of a group with the property that two given members are either in a relationship or not [13]. Relationship such as communication, power, liking, disliking etc. can be represented by a matrix in which all the elements are either 0 or 1 . Along with its applications in Social networks, cliques are widely used in Bioinformatics. Many different problems from Bioinformatics have been modelled using cliques and clique matrix can be used to formulate these problems. In Electrical Engineering clique matrices are used to analyze communication networks. Clique matrices are applied in Statistics for clustering problems i.e. clique matrix is used to decompose a graph into a set of possibly overlapping clusters defined as a well connected subset of vertices. Clique matrix is also used in Political networks and Democratic networks, where cliques are made of symmetric binary relations and clique membership (or Clique affiliation) matrix which describes if a node is a member of a clique or not.

\section{CONCLUSION}

In this paper, we have used clique matrix, which is a generalization of the incidence matrix to study traffic control problem at an intersection is an efficient graph theoretic tool to obtain a solution to the control problem. Here we have shown a simple example with five streams but the idea can be generalized for a larger intersection where the numbers of streams are more as a result of which the number of cliques formed will also be more. To find a solution to the control problem from the clique matrix, phasing can be done from the clique matrix itself and thus offering a useful tool to control traffic at an intersection.

\section{ACKNOWLEDGEMENTS}

This Research is funded by grants from the UGC, New Delhi, India as a Major Research Project awarded to Arun Kumar Baruah. Niky Baruah is associated with the project as a Project Fellow.

\section{REFERENCES}

[1] Gazis, D. C., 1970, Traffic Science, John Wiley \& Sons, New York.

[2] Gazis, D. C., 2002, Traffic Theory, Kluwer Academic Publishers, London.

[3] Guberinic, S., Senborn, G., Lazic, B., 2008, Optimal Traffic Control : Urban Intersection, CRC Press.

[4] Review of Road Traffic Control Strategies : Proceeding of the IEEE, Vol. 91, No.12, December 2003

[5] Barber, D., 2008, Clique Matrices for Statistical Graph Decomposition and Parameterising Restricted Positive Definite Matrices, Uncertainity in Artificial Intelligence.

[6] Deo, N., 2002, Graph Theory with Applications to Engineering and Computer Science, Prentice Hall of India.

[7] Kumar, A., Athisayanathan, S., Antonysamy, A., 2010 Algorithm to Find All Cliques in a Graph, International Journal of Advanced Networking and Applications, Vol. 02, Issue 02, p. 597-601.

[8] Augustson, J .G.,. Minker, J., 1970, An analysis of some graph theoretical cluster techniques, Journal of ACM, Vol. 17, No. 4, p. 571-588.

[9] Mitten, L. G., Branch and Bound Methods : General Formulation and Properties, 1970, Opns. Res., Vol. 18, p. 24-34

[10] Coen Bron and Joep Kerboscht, 1937, Finding All Cliques of an Undirected Graph[H], Communications of the ACM, Vol. 16, No. 9, p. 575 - 580.

[11] Baruah, A. K., Baruah, N., 2012, Signal Groups of Compatible Graph in Traffic Control Problems, International Journal of Advanced Networking and Applications, Vol. 04, Issue 01, p. 1473-1480.

[12] Chartrand, G., 1977, Introductory Graph Theory, Dover Publishers, Inc., New York.

[13] Roberts, F. S., Graph Theory and Its Application to Social Science, 1978, Regional Conference Series in Applied Mathematics. 